A novel numerical mechanical model for the stress-strain distribution in superconducting cable-in-conduit conductors

This article has been downloaded from IOPscience. Please scroll down to see the full text article.

2011 Supercond. Sci. Technol. 24065012

(http://iopscience.iop.org/0953-2048/24/6/065012)

View the table of contents for this issue, or go to the journal homepage for more

Download details:

IP Address: 130.89.101.220

The article was downloaded on 05/12/2011 at 18:09

Please note that terms and conditions apply. 


\title{
A novel numerical mechanical model for the stress-strain distribution in superconducting cable-in-conduit conductors
}

\author{
J Qin ${ }^{1,2}$, Y Wu ${ }^{1}$, L L Warnet ${ }^{3}$ and A Nijhuis ${ }^{2}$ \\ ${ }^{1}$ Institute of Plasma Physics, Chinese Academy of Sciences, Hefei, Anhui 230031, \\ People's Republic of China \\ ${ }^{2}$ Energy, Materials and Systems, Faculty of Science and Technology, University of Twente, \\ Enschede, 7500AE, The Netherlands \\ ${ }^{3}$ Division of Design, Production and Manufacturing, Faculty of Mechanical Engineering, \\ University of Twente, Enschede, $7500 \mathrm{AE}$, The Netherlands
}

Received 17 December 2010, in final form 15 March 2011

Published 7 April 2011

Online at stacks.iop.org/SUST/24/065012

\begin{abstract}
Besides the temperature and magnetic field, the strain and stress state of the superconducting $\mathrm{Nb}_{3} \mathrm{Sn}$ wires in multi-stage twisted cable-in-conduit conductors (CICCs), as applied in ITER or high field magnets, strongly influence their transport properties. For an accurate quantitative prediction of the performance and a proper understanding of the underlying phenomena, a detailed analysis of the strain distribution along all individual wires is required. For this, the thermal contraction of the different components and the huge electromagnetic forces imposing bending and contact deformation must be taken into account, following the complex strand pattern and mutual interaction by contacts from surrounding strands. In this paper, we describe a numerical model for a superconducting cable, which can simulate the strain and stress states of all single wires including interstrand contact force and associated deformation. The strands in the cable can be all similar $\left(\mathrm{Nb}_{3} \mathrm{Sn} / \mathrm{Cu}\right)$ or with the inclusion of different strand materials for protection ( $\mathrm{Cu}$, Glidcop).

The simulation results are essential for the analysis and conductor design optimization from cabling to final magnet operation conditions. Comparisons are presented concerning the influence of the sequential cable twist pitches and the inclusion of copper strands on the mechanical properties and thus on the eventual strain distribution in the $\mathrm{Nb}_{3} \mathrm{Sn}$ filaments when subjected to electromagnetic forces, axial force and twist moment. Recommendations are given for conductor design improvements.
\end{abstract}

(Some figures in this article are in colour only in the electronic version)

\section{Introduction}

ITER is a joint international research and development project $[1,2]$ that aims to demonstrate the scientific and technical feasibility of fusion power. The ITER magnet system is made up of four main sub-systems: the 18 toroidal field coils, referred to as TF coils; the central solenoid coils, referred to as CS coils; the six poloidal field coils, referred to as PF coils; and the correction coils, referred to as CCs. All coils with different dimensions used cable-in-conduit conductors (referred to as
CICCs) in their turn have different layouts. The key point in a CICC is the superconductor cable. The cable of more than 1000 strands is build up from different cabling stages with a diameter of $0.8 \mathrm{~mm}$ and a void fraction of $30 \%$ for optimal helium cooling, see figure 1 .

The cable is enclosed in a (stainless steel) conduit and a helium cooling channel in the axial center allows a low pressure drop of the helium flow through the large CICC sections in the coils. Already in the phase of cable manufacturing, the strands are subjected to stress in order to 


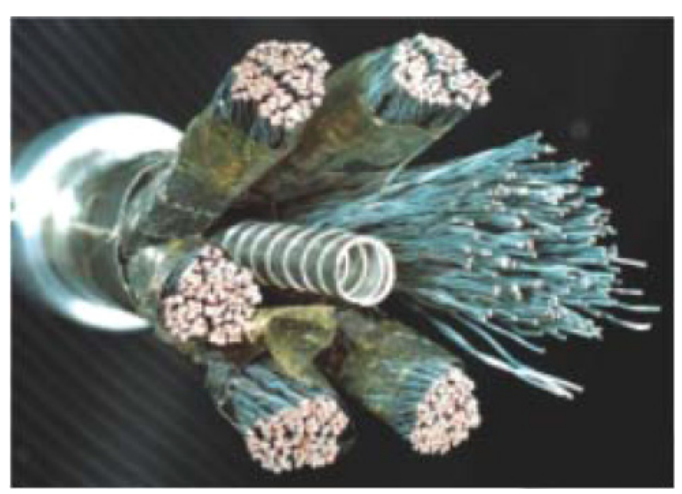

Figure 1. Layout of an ITER CICC.

control the cabling process and, in addition, stress and strain distributions are created in the strands. The stress level for cabling is important to guarantee a good quality cabling pattern but, at the same time, too high stresses can affect the transport properties. In particular for the application of $\mathrm{Nb}_{3} \mathrm{Sn}$ strands (CS and TF), the strain condition of the $\mathrm{Nb}_{3} \mathrm{Sn}$ layers is crucial, as the current transport performance of this superconductor is very sensitive to strain. After the reaction of the $\mathrm{Nb}_{3} \mathrm{Sn}$ layers during a heat treatment at more than $900 \mathrm{~K}$, the CICC is cooled to its operating temperature of $5 \mathrm{~K}$. As the strands in the cable and the conduit have a different thermal contraction, the strands are subjected to cool-down strain. When the coil is charged with current, the electromagnetic Lorentz forces create a transverse load on the cable and in addition hoop stress causes axial tensile stress. Altogether, the strands in the cable are subjected to axial force, twist moment, bending moment, thermal contraction and Lorentz force during cabling and low temperature operating conditions. The necessary condition for superconductor cable operation is the maintenance of temperature in a narrow range of temperature between the helium inlet cooling temperature of about $4.5 \mathrm{~K}$ and the CICC current sharing temperature $\left(T_{\mathrm{cs}}\right)$ of about $7 \mathrm{~K}$. Above the $T_{\mathrm{cs}}$ the CICC becomes normal and the coil quenches, which can be reached by heat generation under pulsed loading of the conductors. Another important aspect that limits the $T_{\mathrm{cs}}$ is the performance degradation due to excessive accumulated deformations and residual strains initiated during cool down and charging of the coils, creating transverse loads on the strand layers in the CICC. The strain state of, in particular, the superconducting $\mathrm{Nb}_{3} \mathrm{Sn}$ wires in multi-stage twisted ITER CICCs strongly determines the transport properties [3, 4]. And so, for an accurate prediction of the performance and a proper understanding of the underlying phenomena, a detailed analysis of the stress and strain distributions along all individual wires is imperative.

The starting point for our analysis is that the superconducting cable structure is similar to a wire rope. A complex wire rope consists of several helical strands. Each strand may be composed of several wires or several strands. Most previous researches focused on the mechanics of a wire rope only analyzed the axially loaded case [5, 7-18]. Most theory is based on the general theorem of thin rods [6], including Costello and Velinsky's model [6-8], Lee's model [10],
Raoof's model [11] and Yen's model [12]. Costello's and Raoof's model is concerned with the whole rope, and assumes every stage of a rope as a whole. In this case it is difficult to analyze the stress of a single wire. Lee's model used Cartesian coordinate equations to describe the helical geometry of wires within a rope. The curvature and torsion can be obtained from the three-dimensional geometry described by the Frenet formulas. However, the model cannot be used to analyze the contact deformation and stress. The theory of the strand was therefore extended to analyze axially loaded complex wire ropes in Yen's model, in which individual wires were considered as slender rods. The loads acting on each wire include the contact force and friction and these were determined based on the various contact patterns among the wires. Yen's model provides the curvature components by an analogous concept, which is the relative rotational motion of particles around fixed and moving axes. Accordingly this is combined with the use of Love's thin rods theory. However, this combination causes a conflict with one of the equilibrium equations of the thin wire theory and for this reason finite element models based on the elastic theory have been proposed [16, 18]. Nemov et al used two models to solve the problem of determining the superconducting cable stress-strain state under tensile and twisting loads [19]. The first approach is based on the theory of rope. The second approach is to solve the general elasticity theory equations with appropriate boundary conditions. In the two models, the strand is supposed to be homogeneous and isotropic with a constant Young's modulus.

A new mechanical model for a superconducting cable (CORD) based on wire rope theory [5, 6, 20, 21] is developed and described in this paper. The model can predict the local strain and stress state of all individual wires, including interstrand contact force and the associated deformation. The tangent modulus is used, which conforms to the experimentally determined axial stress-strain curve. In section 2, we give the general descriptions of relative parameters and rod theory. In section 3, we propose an accurate model for a triplet (first cabling stage existing out of three strands) loaded with axial force and torsion for which the contact deformation is also considered in the model. In section 4 , we extend the triplet model to stage 2 and stage 3 . In section 5 , we present the model results and comparison to the experiments. We discuss the influence of the sequential cable twist pitches and the inclusion of separate copper strands (segregated copper) on the mechanical properties of the cable. The model can provide the approximate stress and strain distribution in a cable. The simulation results are not only important for analysis but may even be more essential for conductor design optimization. This paper aims in providing a full description of the CORD model while most results obtained so far have been reported in [23].

\section{Basic definitions}

\subsection{Coordinate systems and notations}

A Cartesian coordinate system $(X-Y-Z)$ is established, where the $Z$-axis coincides with the center line of the cable. The local coordinate system is formed by a Frenet frame $(p-b-t)$ with 


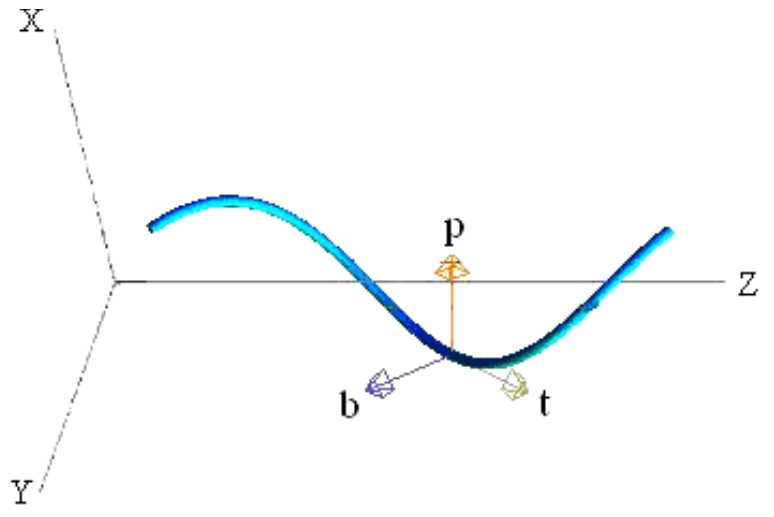

Figure 2. Coordinate systems.

Table 1. List of symbols.

\begin{tabular}{ll}
\hline$R$ & Radius of wire \\
$E$ & Young's modulus of wire \\
$v$ & Poisson ratio of wire \\
$A_{0}$ & Cross section of single wire \\
$p_{i}$ & Twist pitch of the $i$ th stage of cable \\
$\alpha_{i}$ & Twist angle of the $i$ th stage of cable \\
$\epsilon_{0}$ & Single wire strain \\
$\epsilon_{i}$ & Strain of the $i$ th stage of cable \\
$\gamma_{i}$ & Twist per unit length of the $i$ th stage of cable \\
$r_{i}$ & Twist radius of the $i$ th stage of cable \\
$D_{i}$ & Diameter of the $i$ th stage of cable \\
$\xi_{i}$ & Twist starting angle of the $i$ th stage of cable \\
$\delta$ & Contact deformation function \\
$\kappa_{\mathrm{p}_{0}}, \kappa_{\mathrm{b}_{0}}, \kappa_{\mathrm{t}_{0}}$ & Curvature components of single wire \\
$\kappa_{\mathrm{p}_{i}}, \kappa_{\mathrm{b}_{i}}, \kappa_{\mathrm{t}_{i}}$ & Curvature components of the $i$ th stage of cable \\
\hline
\end{tabular}

unit principal normal, binormal, and tangent vectors, shown in figure 2 . The symbols used in this paper are defined in table 1 .

\subsection{Thin rod theory}

For the convenience of the reader, this section reviews the thin rod theory $[5,6]$.

Consider a thin wire loaded with the force shown in figure 3. Let $s$ be the arc length along the wire. $F_{\mathrm{p}}, F_{\mathrm{b}}$, and $F_{\mathrm{t}}$ are sectional force components of the wire, and $M_{\mathrm{p}}, M_{\mathrm{b}}$, and $M_{\mathrm{t}}$ are sectional moment components of the wire. $F_{x}, F_{y}$, and $F_{z}$ are the components of the external line load, and $M_{x}, M_{y}$, and $M_{z}$ are the components of the external moment.

The equilibriums for the loaded thin rod can be obtained from $[5,6]$

$$
\begin{gathered}
\frac{\mathrm{d} F_{\mathrm{p}}}{\mathrm{d} s}-F_{\mathrm{b}} \kappa_{\mathrm{t}}+F_{\mathrm{t}} \kappa_{\mathrm{b}}+F_{x}=0, \\
\frac{\mathrm{d} F_{\mathrm{b}}}{\mathrm{d} s}-F_{\mathrm{t}} \kappa_{\mathrm{p}}+F_{\mathrm{p}} \kappa_{\mathrm{t}}+F_{y}=0, \\
\frac{\mathrm{d} F_{\mathrm{t}}}{\mathrm{d} s}-F_{\mathrm{p}} \kappa_{\mathrm{b}}+F_{\mathrm{b}} \kappa_{\mathrm{p}}+F_{z}=0, \\
\frac{\mathrm{d} F_{\mathrm{p}}}{\mathrm{d} s}-M_{\mathrm{b}} \kappa_{\mathrm{t}}+M_{\mathrm{t}} \kappa_{\mathrm{b}}-F_{\mathrm{b}}+M_{x}=0, \\
\frac{\mathrm{d} M_{\mathrm{b}}}{\mathrm{d} s}-M_{\mathrm{t}} \kappa_{\mathrm{p}}+M_{\mathrm{p}} \kappa_{\mathrm{t}}+F_{\mathrm{p}}+M_{y}=0,
\end{gathered}
$$

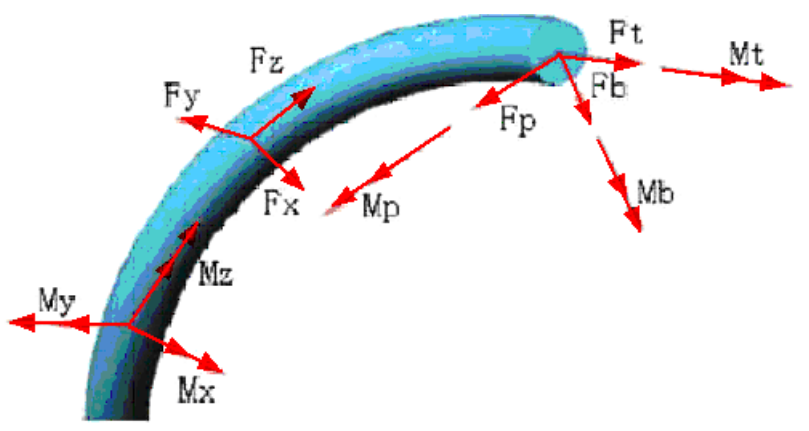

Figure 3. Loading acting on rod.

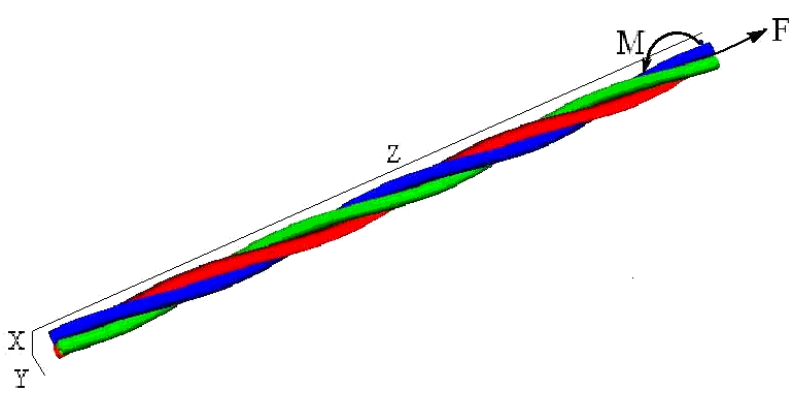

Figure 4. Triplet under axial force and torsion.

$$
\frac{\mathrm{d} M_{\mathrm{t}}}{\mathrm{d} s}-M_{\mathrm{p}} \kappa_{\mathrm{b}}+M_{\mathrm{b}} \kappa_{\mathrm{p}}+M_{z}=0,
$$

where $\kappa_{\mathrm{p}}, \kappa_{\mathrm{b}}$, and $\kappa_{\mathrm{t}}$ are curvature components.

\section{Triplet model}

In this section, we provide a description of the triplet model and the functions for twist radius and contact deformation.

\subsection{Triplet model under axial force and torsion}

Here, we give the model for applying wires of different materials in a triplet, which is under axial force and torsion (figure 4).

In the model, we assume that the diameters of the materials in the triplet are the same. First, we give the parameter equations of the single wire:

$$
\begin{gathered}
X=r_{1} \cos \theta, \\
Y=r_{1} \sin \theta, \\
Z=r_{1} \theta \tan \alpha_{1},
\end{gathered}
$$

where $\alpha_{1}=\arctan \frac{2 \pi r_{1}}{p_{1}}, \alpha_{1} \in\left(0, \frac{\pi}{2}\right)$.

The process for contact deformation between wires is illustrated in figure 5 and the relative equations for the contact force in equilibrium with the force for the first wire $\left(O_{1}\right)$ can be written as follows:

$$
\begin{gathered}
F_{x_{1}}=X_{\mathrm{c}_{1}} \cos \bar{\psi}_{11}+Y_{\mathrm{c}_{1}} \cos \bar{\psi}_{12}, \\
F_{y_{1}}=X_{\mathrm{c}_{1}} \sin \bar{\psi}_{11}-Y_{\mathrm{c}_{1}} \sin \bar{\psi}_{12} .
\end{gathered}
$$




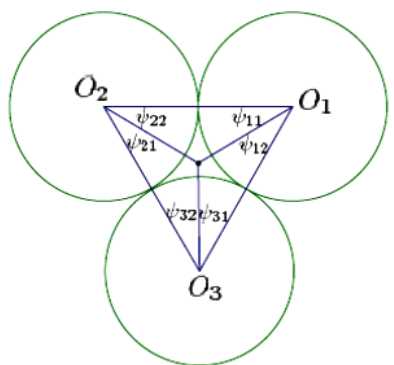

(a)

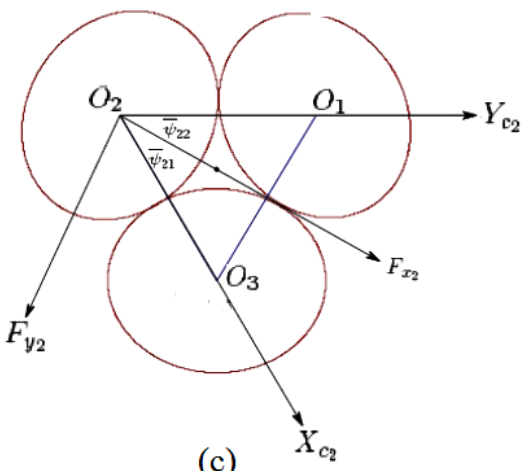

(c)
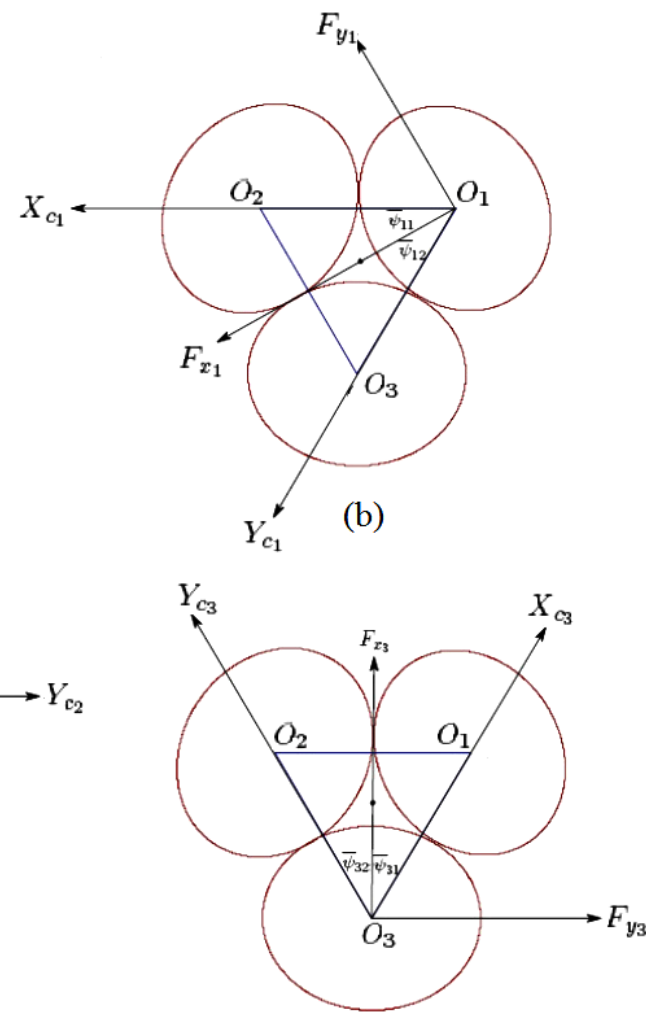

(d)

Figure 5. Contact between wires.

The same equations can be obtained for $\mathrm{O}_{2}$ and $\mathrm{O}_{3}$ :

$$
\begin{aligned}
& F_{x_{2}}=X_{\mathrm{c}_{2}} \cos \bar{\psi}_{21}+Y_{\mathrm{c}_{2}} \cos \bar{\psi}_{22}, \\
& F_{y_{2}}=X_{\mathrm{c}_{2}} \sin \bar{\psi}_{21}-Y_{\mathrm{c}_{2}} \sin \bar{\psi}_{22}, \\
& F_{x_{3}}=X_{\mathrm{c}_{3}} \cos \bar{\psi}_{31}+Y_{\mathrm{c}_{3}} \cos \bar{\psi}_{32}, \\
& F_{y_{3}}=X_{\mathrm{c}_{3}} \sin \bar{\psi}_{31}-Y_{\mathrm{c}_{3}} \sin \bar{\psi}_{32},
\end{aligned}
$$

where $X_{\mathrm{c}_{1}}, Y_{\mathrm{c}_{1}}, X_{\mathrm{c}_{2}}, Y_{\mathrm{c}_{2}}, X_{\mathrm{c}_{3}}$, and $Y_{\mathrm{c}_{3}}$ are contact forces. The external forces $F_{y_{1}}, F_{y_{2}}$, and $F_{y_{3}}$ are 0 when triplet is under axial force or twist moment. From the above equations and the relations of contact forces, we can get the following equalities:

$$
X_{\mathrm{c}_{1}}=Y_{\mathrm{c}_{2}}, \quad X_{\mathrm{c}_{2}}=Y_{\mathrm{c}_{3}}, \quad X_{\mathrm{c}_{3}}=Y_{\mathrm{c}_{1}} \text {. }
$$

From this, we can get the system of equations:

$$
\begin{aligned}
& F_{x_{1}} \sin \bar{\psi}_{12}=X_{\mathrm{c}_{1}} \sin \left(\bar{\psi}_{11}+\bar{\psi}_{12}\right), \\
& F_{x_{2}} \sin \bar{\psi}_{22}=X_{\mathrm{c}_{2}} \sin \left(\bar{\psi}_{21}+\bar{\psi}_{22}\right), \\
& F_{x_{3}} \sin \bar{\psi}_{32}=X_{\mathrm{c}_{3}} \sin \left(\bar{\psi}_{31}+\bar{\psi}_{32}\right) .
\end{aligned}
$$

Meanwhile, the resultant force $\left(F_{e}\right)$ of $F_{x_{1}}, F_{x_{2}}$ and $F_{x_{3}}$ should be 0 , written as

$$
F_{e}\left(F_{x_{1}}, F_{x_{2}}, F_{x_{3}}\right)=0 .
$$

The relative equation about angles is

$$
\bar{\psi}_{11}+\bar{\psi}_{12}+\bar{\psi}_{21}+\bar{\psi}_{22}+\bar{\psi}_{31}+\bar{\psi}_{32}=2 \pi \text {. }
$$
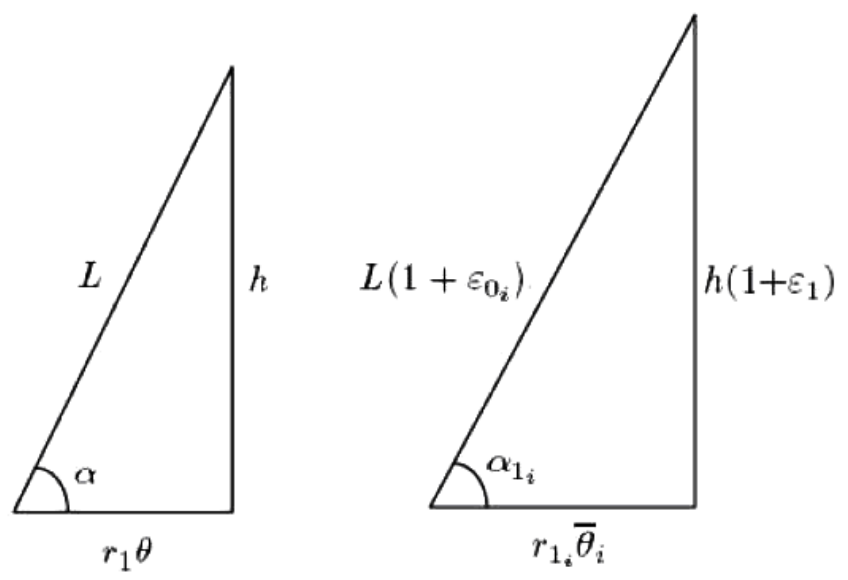

Figure 6. Developed view of the single wire.

Now, we give the mathematical model. The developed view of the single wire in the triplet is plotted in figure 6 . The axial strain of the triplet is defined as $\epsilon_{1}$, and the rotational strain of one single wire is defined as

$$
\gamma_{1}=r_{1} \frac{\bar{\theta}_{i}-\theta}{h}
$$

Using some relations of angles and edges in the triangles, we can obtain the relation equations from figure 6 as

$$
\epsilon_{1}=\left(1+\epsilon_{0_{i}}\right) \frac{\sin \bar{\alpha}_{1_{i}}}{\sin \alpha_{1}}-1,
$$



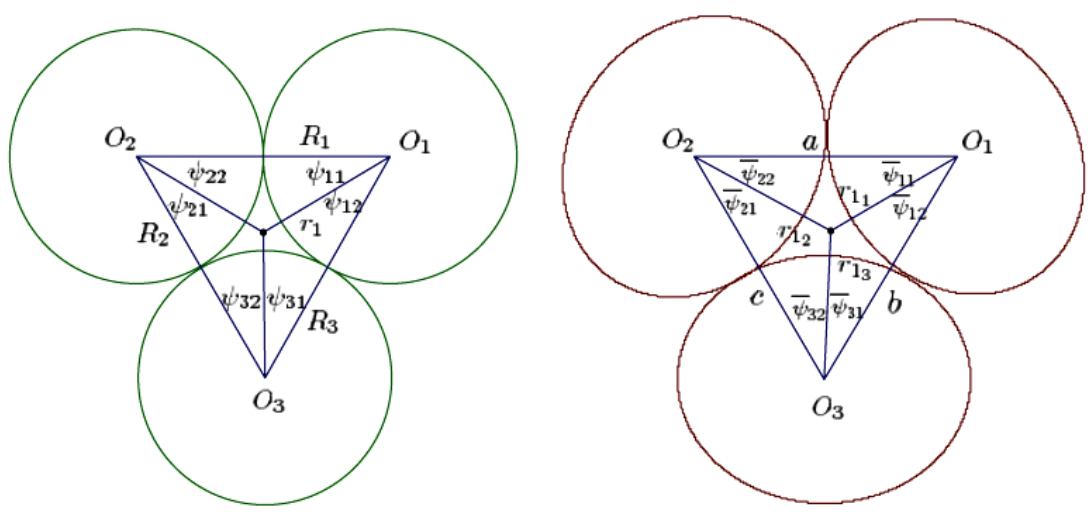

Figure 7. Contact between wires (left: before contact deformation; right: after contact deformation),

$$
\gamma_{1}=\frac{r_{1}}{\bar{r}_{1_{i}}} \frac{1+\epsilon_{1}}{\tan \bar{\alpha}_{1_{i}}}-\frac{1}{\tan \alpha_{1}} .
$$

The original components of the curvature and the twist per unit length are

$$
\kappa_{\mathrm{p}_{0}}=0, \quad \kappa_{\mathrm{b}_{0}}=\frac{\cos ^{2} \alpha_{1}}{r_{1}}, \quad \kappa_{\mathrm{t}_{0}}=\frac{\sin \alpha_{1} \cos \alpha_{1}}{r_{1}} .
$$

The components of the curvature and the twist per unit length of every wire under loading are

$$
\bar{\kappa}_{\mathrm{p}_{0_{i}}}=0, \quad \bar{\kappa}_{\mathrm{b}_{0_{i}}}=\frac{\cos ^{2} \bar{\alpha}_{1_{i}}}{\bar{r}_{1_{i}}}, \quad \bar{\kappa}_{\mathrm{t}_{0_{i}}}=\frac{\sin \bar{\alpha}_{1_{i}} \cos \bar{\alpha}_{1}}{\bar{r}_{1_{i}}} .
$$

In the analysis, a wire is regarded as a thin rod, and the sectional moments are related to the changes of curvature and torsion. The moments of a single wire can be obtained with

$$
\begin{aligned}
M_{\mathrm{p}_{i}} & =E_{i} I_{\mathrm{p}_{i}}\left(\bar{\kappa}_{\mathrm{p}_{0_{i}}}-\kappa_{\mathrm{p}_{0}}\right), \\
M_{\mathrm{b}_{i}} & =E_{i} I_{\mathrm{b}_{i}}\left(\bar{\kappa}_{\mathrm{b}_{0_{i}}}-\kappa_{\mathrm{b}_{0}}\right), \\
M_{\mathrm{t}_{i}} & =E_{i} I_{\mathrm{t}_{i}}\left(\bar{\kappa}_{\mathrm{t}_{0_{i}}}-\kappa_{\mathrm{t}_{0}}\right) .
\end{aligned}
$$

The axial force in the single wire is given by

$$
F_{\mathrm{t}_{i}}=A_{0} \sigma_{i}\left(\epsilon_{0_{i}}\right) \text {. }
$$

According to the above equilibrium of a thin wire, the sectional shear forces and contact force can be expressed by

$$
\begin{gathered}
F_{\mathrm{p}_{i}}=-\frac{\mathrm{d} M_{\mathrm{b}_{i}}}{\mathrm{~d} s}+M_{\mathrm{t}_{i}} \cdot \kappa_{\mathrm{p}_{0_{i}}}-M_{\mathrm{p}_{i}} \cdot \kappa_{\mathrm{t}_{0_{i}}}, \\
F_{\mathrm{b}_{i}}=\frac{\mathrm{d} M_{\mathrm{p}_{i}}}{\mathrm{~d} s}-M_{\mathrm{b}_{i}} \cdot \kappa_{\mathrm{t}_{0_{i}}}+M_{\mathrm{t}_{i}} \cdot \kappa_{\mathrm{b}_{0_{i}}}, \\
F_{x_{i}}=F_{\mathrm{b}_{i}} \cdot \kappa_{\mathrm{t}_{0_{i}}}-F_{\mathrm{t}_{i}} \cdot \kappa_{\mathrm{b}_{0_{i}}} .
\end{gathered}
$$

The total axial force $F$ and the total axial twisting moment $M$ acting on the triplet can be obtained by

$$
F=\Sigma_{i=1}^{3}\left(F_{\mathrm{t}_{i}} \sin \alpha_{1}+F_{\mathrm{b}_{i}} \cos \alpha_{1}\right),
$$

$M=\Sigma_{i=1}^{3}\left(M_{\mathrm{t}_{i}} \sin \alpha_{1}+M_{\mathrm{b}_{i}} \cos \alpha_{1}+F_{\mathrm{t}_{i}} r_{1} \cos \alpha_{1}-F_{\mathrm{b}_{i}} r_{1} \sin \alpha_{1}\right)$.

There are nineteen unknown quantities in the model, which are $F, M, X_{\mathrm{c}_{1}}, X_{\mathrm{c}_{2}}, X_{\mathrm{c}_{3}}, \bar{\psi}_{11}, \bar{\psi}_{12}, \bar{\psi}_{21}, \bar{\psi}_{22}, \bar{\psi}_{31}, \bar{\psi}_{32}$, $\epsilon_{1}, \epsilon_{0_{1}}, \epsilon_{0_{2}}, \epsilon_{0_{3}}, \gamma_{1}, \bar{\alpha}_{1_{1}}, \bar{\alpha}_{1_{2}}$, and $\bar{\alpha}_{1_{3}}$. The relative equations are equations (17)-(24), equations (32) and (33) and some triangle relationship equations. The system of equations is nonlinear. Therefore, we apply the Newton method to solve it when we know the values of two quantities. Generally, we solve the system of equations with known axial force $(F)$ and twist moment $(M)$ or $\epsilon_{1}$ and $\gamma_{1}$.

\subsection{Functions of changed twist radius and contact deformation}

In this section, we will give the relative formulas of changed twist radius and contact deformation. From figure 7, we can derive the relative equations

$$
\begin{aligned}
& \frac{r_{1_{1}}}{\sin \bar{\psi}_{22}}=\frac{r_{1_{2}}}{\sin \bar{\psi}_{11}}=\frac{a}{\sin \left(2 \pi-\bar{\psi}_{11}-\bar{\psi}_{22}\right)}, \\
& \frac{r_{1_{2}}}{\sin \bar{\psi}_{32}}=\frac{r_{1_{3}}}{\sin \bar{\psi}_{21}}=\frac{a}{\sin \left(2 \pi-\bar{\psi}_{21}-\bar{\psi}_{32}\right)}, \\
& \frac{r_{1_{3}}}{\sin \bar{\psi}_{12}}=\frac{r_{1_{1}}}{\sin \bar{\psi}_{31}}=\frac{a}{\sin \left(2 \pi-\bar{\psi}_{12}-\bar{\psi}_{31}\right)}, \\
& a=R_{1}\left(1-v_{1} \epsilon_{0_{1}}\right)+R_{2}\left(1-v_{2} \epsilon_{0_{2}}\right)-\delta_{1}, \\
& b=R_{1}\left(1-v_{1} \epsilon_{0_{1}}\right)+R_{3}\left(1-v_{3} \epsilon_{0_{3}}\right)-\delta_{2}, \\
& c=R_{2}\left(1-v_{2} \epsilon_{0_{2}}\right)+R_{3}\left(1-v_{3} \epsilon_{0_{3}}\right)-\delta_{3} .
\end{aligned}
$$

The function describing the contact deformation $\left(\delta_{1}\right)$ can be written as follows:

$$
\begin{aligned}
\delta_{1}= & \frac{X_{\mathrm{f}}}{\pi}\left[\frac{1-v_{1}^{2}}{E_{1}\left(\epsilon_{0_{1}}\right)}\left(2 \ln \left(\frac{4 R_{1}}{B}\right)-1\right)\right. \\
& \left.+\frac{1-v_{2}^{2}}{E_{2}\left(\epsilon_{0_{2}}\right)}\left(2 \ln \left(\frac{4 R_{2}}{B}\right)-1\right)\right], \\
B & =\sqrt{\frac{2 X_{\mathrm{f}} \Delta}{\pi}} \\
X_{\mathrm{f}} & =-X_{c i}, \\
\Delta & =\frac{2 R_{1} R_{2}}{R_{1}+R_{2}}\left(\frac{1-v_{1}^{2}}{E_{1}\left(\epsilon_{0_{1}}\right)}+\frac{1-v_{2}^{2}}{E_{2}\left(\epsilon_{0_{2}}\right)}\right) .
\end{aligned}
$$

The functions $\delta_{2}$ and $\delta_{3}$ are similar to $\delta_{1}$. 


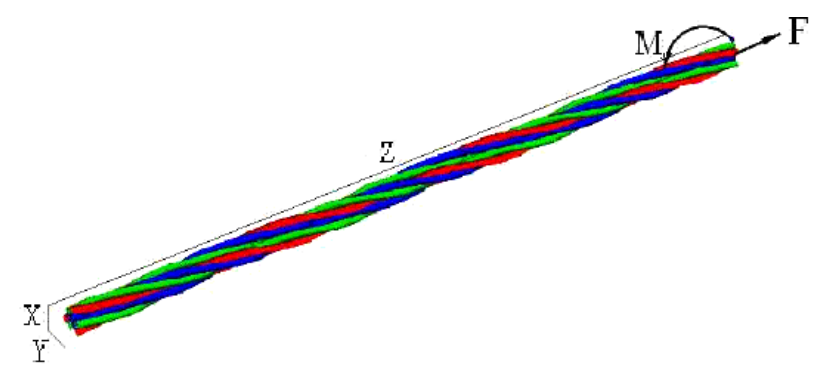

Figure 8. Second stage under axial force and torsion

\section{The model for the second stage and third stage}

In this section, we present the models for stages 2 and 3 for which an analogous approach is followed.

\subsection{The model for the second stage}

In this section, we introduce the model for the second stage under axial force and torsion (figure 8).

The single wire is a second-order helical line, and the triplet is a first-order helical line within the second stage, as shown in figure 9. We first get the curvature components of the triplet and single wire from the matrix given in [12] as

$T_{1}=$

$\left(\begin{array}{ccc}-\cos \left(\theta_{1}+\xi_{1}\right) & -\sin \left(\theta_{1}+\xi_{1}\right) & 0 \\ \sin \alpha_{1} \sin \left(\theta_{1}+\xi_{1}\right) & -\sin \alpha_{1} \cos \left(\theta_{1}+\xi_{1}\right) & \cos \alpha_{1} \\ -\cos \alpha_{1} \sin \left(\theta_{1}+\xi_{1}\right) & \cos \alpha_{1} \cos \left(\theta_{1}+\xi_{1}\right) & \sin \alpha_{1}\end{array}\right)$,

$T_{2}=$

$\left(\begin{array}{ccc}-\cos \left(\theta_{2}+\xi_{2}\right) & -\sin \left(\theta_{2}+\xi_{2}\right) & 0 \\ \sin \alpha_{2} \sin \left(\theta_{2}+\xi_{2}\right) & -\sin \alpha_{2} \cos \left(\theta_{2}+\xi_{2}\right) & \cos \alpha_{2} \\ -\cos \alpha_{2} \sin \left(\theta_{2}+\xi_{2}\right) & \cos \alpha_{2} \cos \left(\theta_{2}+\xi_{2}\right) & \sin \alpha_{2}\end{array}\right)$,

$\left(\begin{array}{c}\kappa_{\mathrm{p}_{1}} \\ \kappa_{\mathrm{b}_{1}} \\ \kappa_{\mathrm{t}_{1}}\end{array}\right)=T_{2}\left(\begin{array}{c}0 \\ 0 \\ \frac{\cos \alpha_{2}}{r_{2}}\end{array}\right)$

$\left(\begin{array}{c}\kappa_{\mathrm{p}_{0}} \\ \kappa_{\mathrm{b}_{0}} \\ \kappa_{\mathrm{t}_{0}}\end{array}\right)=T_{1} T_{2}\left(\begin{array}{c}-\frac{\cos \alpha_{1} \cos \alpha_{2} \sin \left(\theta_{2}+\xi_{2}\right)}{r_{2}} \\ \frac{\cos \alpha_{1} \cos \alpha_{2} \cos \left(\theta_{2}+\xi_{2}\right)}{r_{2}} \\ \frac{\cos \alpha_{2} \sin \alpha_{1}}{r_{1}}+\frac{\cos \alpha_{1} \sin \alpha_{2}}{r_{2}}\end{array}\right)$.

Unwinding the triplet and outer wires, then the geometry between an outer wire and the triplet can be presented in figure 10. When using the trigonometrical relations between the unloaded and loaded states, this leads to

$$
\begin{gathered}
\epsilon_{2}=\left(1+\epsilon_{1}\right) \frac{\sin \overline{\alpha_{2}}}{\sin \alpha_{2}}-1, \\
\epsilon_{1}=\left(1+\epsilon_{0}\right) \frac{\sin \overline{\alpha_{1}}}{\sin \alpha_{1}}-1, \\
\gamma_{2}=\frac{r_{2}}{\overline{r_{2}}} \frac{1+\epsilon_{2}}{\tan \overline{\alpha_{2}}}-\frac{1}{\tan \alpha_{2}}, \\
\gamma_{1}=\frac{r_{1}}{\overline{r_{1}}} \frac{1+\epsilon_{1}}{\tan \overline{\alpha_{1}}}-\frac{1}{\tan \alpha_{1}}=\bar{\kappa}_{\mathrm{t}_{1}}-\kappa_{\mathrm{t}_{1}}, \\
\bar{r}_{1}=r_{1}\left(1-v \epsilon_{0}\right), \\
\bar{r}_{2}=r_{2}+\bar{r}_{1}-r_{1}-v \epsilon_{0} R .
\end{gathered}
$$

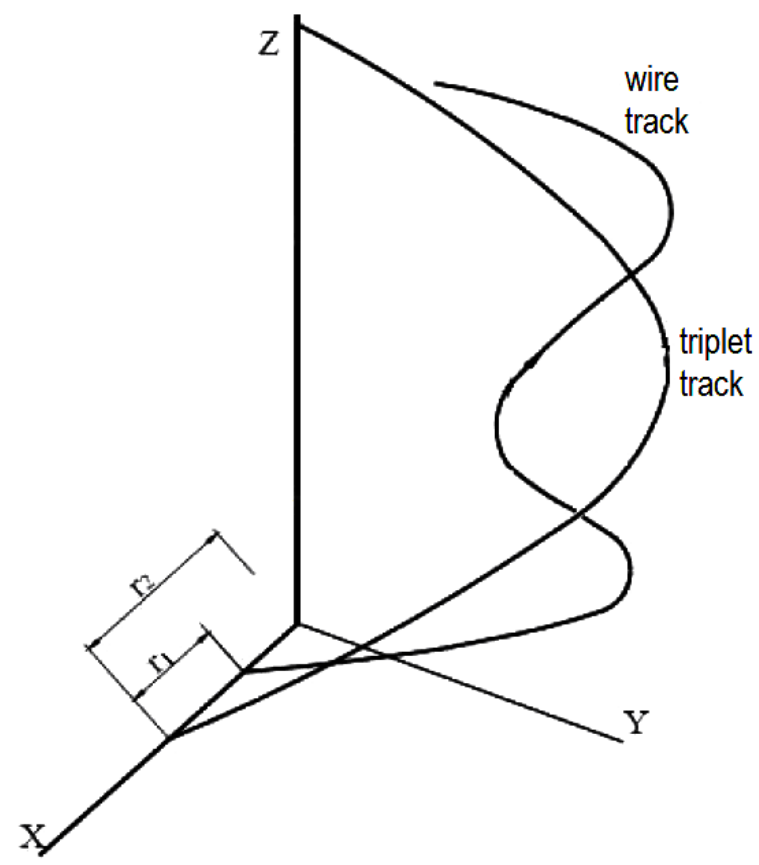

Figure 9. Space line of the triplet and single wire.

In the analysis, a wire is regarded as a thin rod and the sectional moments are related to the changes of curvatures and torsion. The moments of the single wire can be obtained by

$$
\begin{gathered}
M_{\mathrm{p}_{0}}=E I_{\mathrm{p}_{0}}\left(\bar{\kappa}_{\mathrm{p}_{0}}-\kappa_{\mathrm{p}_{0}}\right), \\
M_{\mathrm{b}_{0}}=E I_{\mathrm{b}_{0}}\left(\bar{\kappa}_{\mathrm{b}_{0}}-\kappa_{\mathrm{b}_{0}}\right), \\
M_{\mathrm{t}_{0}}=E I_{\mathrm{t}_{0}}\left(\bar{\kappa}_{\mathrm{t}_{0}}-\kappa_{\mathrm{t}_{0}}\right) .
\end{gathered}
$$

According to the above equilibrium of a thin wire, the sectional shear forces and axial force can be expressed by

$$
\begin{gathered}
F_{\mathrm{p}_{0}}=-\frac{\mathrm{d} M_{\mathrm{b}}}{\mathrm{d} s}+M_{\mathrm{t}} \cdot \kappa_{\mathrm{p}_{0}}-M_{\mathrm{p}} \cdot \kappa_{\mathrm{t}_{0}}, \\
F_{\mathrm{b}_{0}}=\frac{\mathrm{d} M_{\mathrm{p}}}{\mathrm{d} s}-M_{\mathrm{b}} \cdot \kappa_{\mathrm{t}_{0}}+M_{\mathrm{t}} \cdot \kappa_{\mathrm{b}_{0}}, \\
F_{\mathrm{t}_{0}}=A_{0} \sigma\left(\epsilon_{0}\right) .
\end{gathered}
$$

Let $F_{1}=\left(F_{\mathrm{p}_{1}}, F_{\mathrm{b}_{1}}, F_{\mathrm{t}_{1}}\right)^{\mathrm{T}}$ and $M_{1}=\left(M_{\mathrm{p}_{1}}, M_{\mathrm{b}_{1}}, M_{\mathrm{t}_{1}}\right)^{\mathrm{T}}$ be the equivalent sectional force and moment of the triplet in the second stage. They can be determined by transforming the components of wire forces and moments:

$$
\begin{aligned}
F_{1}= & \left(\begin{array}{l}
F_{\mathrm{p}_{1}} \\
F_{\mathrm{b}_{1}} \\
F_{\mathrm{t}_{1}}
\end{array}\right)=n_{s t} T_{1}\left(\begin{array}{c}
F_{\mathrm{p}_{0}} \\
F_{\mathrm{b}_{0}} \\
F_{\mathrm{t}_{0}}
\end{array}\right)_{s t}+n_{c u} T_{1}\left(\begin{array}{c}
F_{\mathrm{p}_{0}} \\
F_{\mathrm{b}_{0}} \\
F_{\mathrm{t}_{0}}
\end{array}\right)_{c u}, \\
M_{1}= & \left(\begin{array}{c}
M_{\mathrm{p}_{1}} \\
M_{\mathrm{b}_{1}} \\
M_{\mathrm{t}_{1}}
\end{array}\right)=n_{s t}\left[T_{1}\left(\begin{array}{c}
M_{\mathrm{p}_{0}} \\
M_{\mathrm{b}_{0}} \\
M_{\mathrm{t}_{0}}
\end{array}\right)_{s t}\right. \\
& \left.+\left(\begin{array}{c}
r_{1}\left(F_{\mathrm{t}_{0}} \sin \alpha_{1}+F_{\mathrm{b}_{0}} \cos \alpha_{1}\right) \sin \theta_{1} \\
-r_{1}\left(F_{\mathrm{t}_{0}} \sin \alpha_{1}+F_{\mathrm{b}_{0}} \cos \alpha_{1}\right) \cos \theta_{1} \\
r_{1}\left(F_{\mathrm{t}_{0}} \cos \alpha_{1}-F_{\mathrm{b}_{0}} \sin \alpha_{1}\right)
\end{array}\right)_{s t}\right]
\end{aligned}
$$



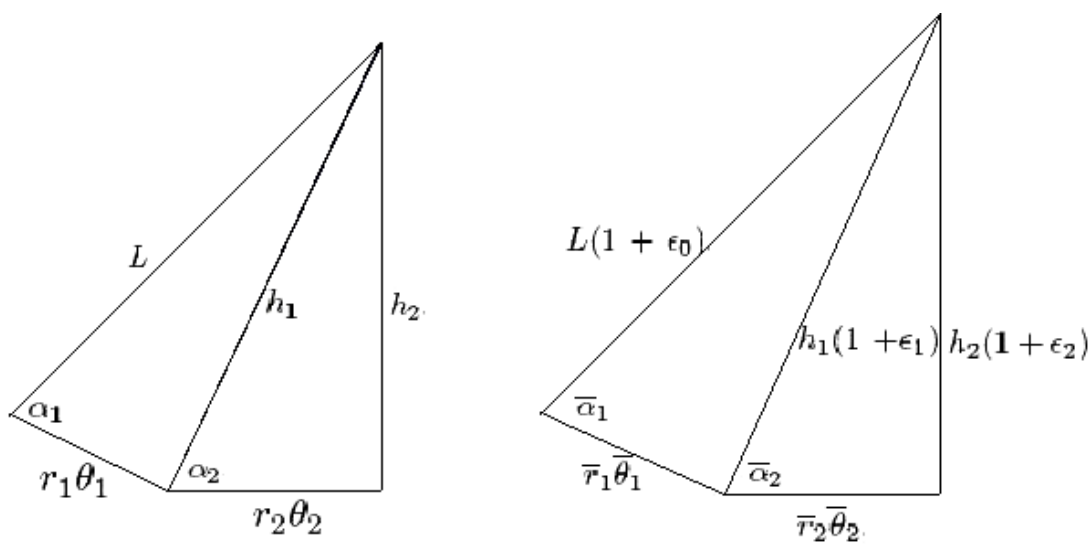

Figure 10. Developed view of the triplet $\left(h_{1}\right)$ and single wire $(L)$.

$$
\begin{aligned}
& +n_{c u}\left[T_{1}\left(\begin{array}{c}
M_{\mathrm{p}_{0}} \\
M_{\mathrm{b}_{0}} \\
M_{\mathrm{t}_{0}}
\end{array}\right)_{c u}\right. \\
& \left.+\left(\begin{array}{c}
r_{1}\left(F_{\mathrm{t}_{0}} \sin \alpha_{1}+F_{\mathrm{b}_{0}} \cos \alpha_{1}\right) \sin \theta_{1} \\
-r_{1}\left(F_{\mathrm{t}_{0}} \sin \alpha_{1}+F_{\mathrm{b}_{0}} \cos \alpha_{1}\right) \cos \theta_{1} \\
r_{1}\left(F_{\mathrm{t}_{0}} \cos \alpha_{1}-F_{\mathrm{b}_{0}} \sin \alpha_{1}\right)
\end{array}\right)_{c u}\right] .
\end{aligned}
$$

Finally, the forces and moments of the second stage can be obtained by transforming the components of the triplet forces and moments:

$$
\begin{aligned}
F_{2}= & \left(\begin{array}{c}
0 \\
0 \\
F_{\mathrm{t}_{2}}
\end{array}\right)=3 T_{2}\left(\begin{array}{l}
F_{\mathrm{p}_{1}} \\
F_{\mathrm{b}_{1}} \\
F_{\mathrm{t}_{1}}
\end{array}\right), \\
M_{2}= & \left(\begin{array}{c}
0 \\
0 \\
M_{\mathrm{t}_{2}}
\end{array}\right)=3\left[T_{2}\left(\begin{array}{l}
M_{\mathrm{p}_{1}} \\
M_{\mathrm{b}_{1}} \\
M_{\mathrm{t}_{1}}
\end{array}\right)\right. \\
& \left.+\left(\begin{array}{c}
r_{2}\left(F_{\mathrm{t}_{1}} \sin \alpha_{2}+F_{\mathrm{b}_{1}} \cos \alpha_{2}\right) \sin \theta_{2} \\
-r_{2}\left(F_{\mathrm{t}_{1}} \sin \alpha_{2}+F_{\mathrm{b}_{1}} \cos \alpha_{2}\right) \cos \theta_{2} \\
r_{2}\left(F_{\mathrm{t}_{1}} \cos \alpha_{2}-F_{\mathrm{b}_{1}} \sin \alpha_{2}\right)
\end{array}\right)\right],
\end{aligned}
$$

where $F_{\mathrm{t}_{2}}$ and $M_{\mathrm{t}_{2}}$ are the axial force and twist moment applied to the second stage, respectively.

\subsection{The model for the third stage}

In this section, we introduce the model for the third stage under axial force and twist moment. The model is analogous to the model describing the second stage cable. The single wire is now a third-order helical line, the triplet is a second-order helical line, and the second stage is a first-order helical line in the third stage.

We first obtain the curvature components of the triplet and single wire by transform matrix:

$$
\begin{aligned}
& T_{1}= \\
& \left(\begin{array}{ccc}
-\cos \left(\theta_{1}+\xi_{1}\right) & -\sin \left(\theta_{1}+\xi_{1}\right) & 0 \\
\sin \alpha_{1} \sin \left(\theta_{1}+\xi_{1}\right) & -\sin \alpha_{1} \cos \left(\theta_{1}+\xi_{1}\right) & \cos \alpha_{1} \\
-\cos \alpha_{1} \sin \left(\theta_{1}+\xi_{1}\right) & \cos \alpha_{1} \cos \left(\theta_{1}+\xi_{1}\right) & \sin \alpha_{1}
\end{array}\right), \\
& T_{2}= \\
& \left.\begin{array}{ccc}
-\cos \left(\theta_{2}+\xi_{2}\right) & -\sin \left(\theta_{2}+\xi_{2}\right) & 0 \\
\sin \alpha_{2} \sin \left(\theta_{2}+\xi_{2}\right) & -\sin \alpha_{2} \cos \left(\theta_{2}+\xi_{2}\right) & \cos \alpha_{2} \\
-\cos \alpha_{2} \sin \left(\theta_{2}+\xi_{2}\right) & \cos \alpha_{2} \cos \left(\theta_{2}+\xi_{2}\right) & \sin \alpha_{2}
\end{array}\right),
\end{aligned}
$$

$$
\begin{aligned}
& T_{3}= \\
& \left(\begin{array}{ccc}
-\cos \left(\theta_{3}+\xi_{3}\right) & -\sin \left(\theta_{3}+\xi_{3}\right) & 0 \\
\sin \alpha_{3} \sin \left(\theta_{3}+\xi_{3}\right) & -\sin \alpha_{3} \cos \left(\theta_{3}+\xi_{3}\right) & \cos \alpha_{3} \\
-\cos \alpha_{3} \sin \left(\theta_{3}+\xi_{3}\right) & \cos \alpha_{3} \cos \left(\theta_{3}+\xi_{3}\right) & \sin \alpha_{3}
\end{array}\right) \\
& \left(\begin{array}{c}
\kappa_{\mathrm{p}_{2}} \\
\kappa_{\mathrm{b}_{2}} \\
\kappa_{\mathrm{t}_{2}}
\end{array}\right)=T_{3}\left(\begin{array}{c}
0 \\
0 \\
\frac{\cos \alpha_{3}}{r_{3}}
\end{array}\right), \\
& \left(\begin{array}{c}
\kappa_{\mathrm{p}_{1}} \\
\kappa_{\mathrm{b}_{1}} \\
\kappa_{\mathrm{t}_{1}}
\end{array}\right)=T_{2} \cdot T_{3}\left(\begin{array}{c}
-\frac{\cos \alpha_{2} \cos \alpha_{3} \sin \left(\theta_{3}+\xi_{3}\right)}{r_{3}} \\
\frac{\cos \alpha_{2} \cos \alpha_{3} \cos \left(\theta_{3}+\xi_{3}\right)}{r_{3}} \\
\frac{\cos \alpha_{3} \sin \alpha_{2}}{r_{2}}+\frac{\cos \alpha_{2} \sin \alpha_{3}}{r_{3}}
\end{array}\right) \\
& \left(\begin{array}{c}
\kappa_{\mathrm{p}_{0}} \\
\kappa_{\mathrm{b}_{0}} \\
\kappa_{\mathrm{t}_{0}}
\end{array}\right)=T_{1} \cdot T_{2} \cdot T_{3} \cdot w,
\end{aligned}
$$

where

$$
\begin{aligned}
w= & T_{3}^{\mathrm{T}} T_{2}^{\mathrm{T}}\left(\begin{array}{c}
0 \\
0 \\
\frac{\cos \alpha_{1}}{r_{1}}
\end{array}\right)+T_{3}^{\mathrm{T}} \sin \alpha_{1}\left(\begin{array}{c}
0 \\
0 \\
\frac{\cos \alpha_{2}}{r_{2}}
\end{array}\right) \\
& +\sin \alpha_{1} \sin \alpha_{2}\left(\begin{array}{c}
0 \\
0 \\
\frac{\cos \alpha_{3}}{r_{3}}
\end{array}\right) .
\end{aligned}
$$

Analogous to the previous case, we unwind the triplet and outer wires, then the geometry between an outer wire and the triplet can be presented as in figure 11. When using the trigonometrical relations between the unloaded and loaded states, we can write

$$
\begin{gathered}
\epsilon_{3}=\left(1+\epsilon_{2}\right) \frac{\sin \bar{\alpha}_{3}}{\sin \alpha_{3}}-1, \\
\epsilon_{2}=\left(1+\epsilon_{1}\right) \frac{\sin \bar{\alpha}_{2}}{\sin \alpha_{2}}-1, \\
\epsilon_{1}=\left(1+\epsilon_{0}\right) \frac{\sin \bar{\alpha}_{1}}{\sin \alpha_{1}}-1, \\
\gamma_{3}=\frac{r_{3}}{\bar{r}_{3}} \frac{1+\epsilon_{3}}{\tan \bar{\alpha}_{3}}-\frac{1}{\tan \alpha_{3}}, \\
\gamma_{2}=\frac{r_{2}}{\bar{r}_{2}} \frac{1+\epsilon_{2}}{\tan \bar{\alpha}_{2}}-\frac{1}{\tan \alpha_{2}}=\bar{\kappa}_{\mathrm{t}_{2}}-\kappa_{\mathrm{t}_{2}}, \\
\gamma_{1}=\frac{r_{1}}{\bar{r}_{1}} \frac{1+\epsilon_{1}}{\tan \bar{\alpha}_{1}}-\frac{1}{\tan \alpha_{1}}=\bar{\kappa}_{\mathrm{t}_{1}}-\kappa_{\mathrm{t}_{1}},
\end{gathered}
$$



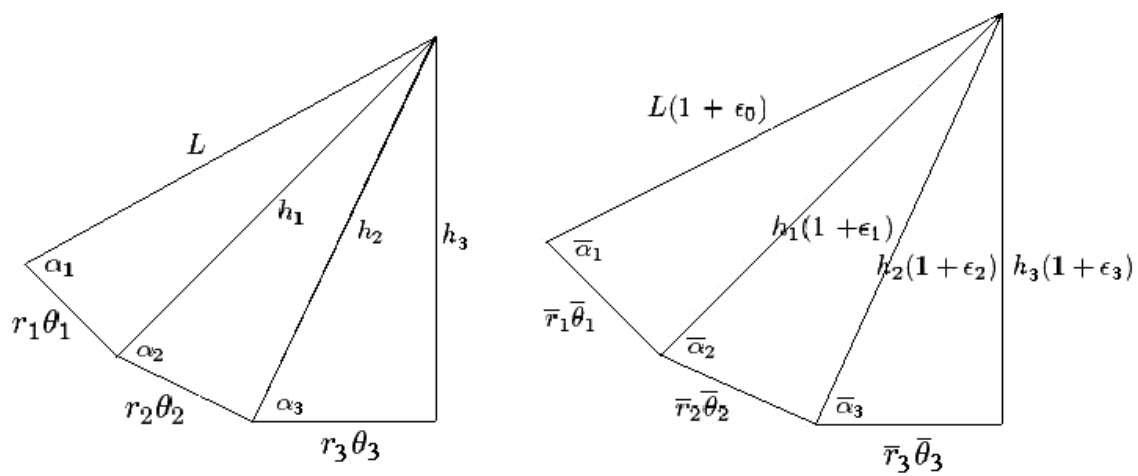

Figure 11. Developed view of the second stage $\left(h_{2}\right)$, triplet $\left(h_{1}\right)$ and single wire $(L)$.

$$
\begin{gathered}
\bar{r}_{1}=r_{1}\left(1-v \epsilon_{0}\right), \\
\bar{r}_{2}=r_{2}+\bar{r}_{1}-r_{1}-v \epsilon_{0} R, \\
\bar{r}_{3}=r_{3}+\bar{r}_{2}-r_{2}-v \epsilon_{0} R .
\end{gathered}
$$

Let $F_{1}=\left(F_{\mathrm{p}_{1}}, F_{\mathrm{b}_{1}}, F_{\mathrm{t}_{1}}\right)^{\mathrm{T}}, M_{1}=\left(M_{\mathrm{p}_{1}}, M_{\mathrm{b}_{1}}, M_{\mathrm{t}_{1}}\right)^{\mathrm{T}}$ and $F_{2}=\left(F_{\mathrm{p}_{2}}, F_{\mathrm{b}_{2}}, F_{\mathrm{t}_{2}}\right)^{\mathrm{T}}, M_{2}=\left(M_{\mathrm{p}_{2}}, M_{\mathrm{b}_{2}}, M_{\mathrm{t}_{2}}\right)^{\mathrm{T}}$ be the equivalent sectional forces and moments of the triplet and second stage, respectively. They can be determined by transforming the components of the wire forces and moments with the same method as applied for the stage 2 model:

$$
\begin{aligned}
& F_{1}=\left(\begin{array}{l}
F_{\mathrm{p}_{1}} \\
F_{\mathrm{b}_{1}} \\
F_{\mathrm{t}_{1}}
\end{array}\right)=n_{s t} T_{1}\left(\begin{array}{l}
F_{\mathrm{p}_{0}} \\
F_{\mathrm{b}_{0}} \\
F_{\mathrm{t}_{0}}
\end{array}\right)_{s t}+n_{c u} T_{1}\left(\begin{array}{c}
F_{\mathrm{p}_{0}} \\
F_{\mathrm{b}_{0}} \\
F_{\mathrm{t}_{0}}
\end{array}\right)_{c u}, \\
& M_{1}=\left(\begin{array}{c}
M_{\mathrm{p}_{1}} \\
M_{\mathrm{b}_{1}} \\
M_{\mathrm{t}_{1}}
\end{array}\right)=n_{s t}\left[T_{1}\left(\begin{array}{c}
M_{\mathrm{p}_{0}} \\
M_{\mathrm{b}_{0}} \\
M_{\mathrm{t}_{0}}
\end{array}\right)_{s t}\right. \\
& \left.+\left(\begin{array}{c}
r_{1}\left(F_{\mathrm{t}_{0}} \sin \alpha_{1}+F_{\mathrm{b}_{0}} \cos \alpha_{1}\right) \sin \theta_{1} \\
-r_{1}\left(F_{\mathrm{t}_{0}} \sin \alpha_{1}+F_{\mathrm{b}_{0}} \cos \alpha_{1}\right) \cos \theta_{1} \\
r_{1}\left(F_{\mathrm{t}_{0}} \cos \alpha_{1}-F_{\mathrm{b}_{0}} \sin \alpha_{1}\right)
\end{array}\right)_{s t}\right] \\
& +n_{c u}\left[T_{1}\left(\begin{array}{c}
M_{\mathrm{p}_{0}} \\
M_{\mathrm{b}_{0}} \\
M_{\mathrm{t}_{0}}
\end{array}\right)_{c u}\right. \\
& \left.+\left(\begin{array}{c}
r_{1}\left(F_{\mathrm{t}_{0}} \sin \alpha_{1}+F_{\mathrm{b}_{0}} \cos \alpha_{1}\right) \sin \theta_{1} \\
-r_{1}\left(F_{\mathrm{t}_{0}} \sin \alpha_{1}+F_{\mathrm{b}_{0}} \cos \alpha_{1}\right) \cos \theta_{1} \\
r_{1}\left(F_{\mathrm{t}_{0}} \cos \alpha_{1}-F_{\mathrm{b}_{0}} \sin \alpha_{1}\right)
\end{array}\right)_{c u}\right], \\
& F_{2}=\left(\begin{array}{c}
F_{\mathrm{p}_{2}} \\
F_{\mathrm{b}_{2}} \\
F_{\mathrm{t}_{2}}
\end{array}\right)=3 T_{1}\left(\begin{array}{c}
F_{\mathrm{p}_{1}} \\
F_{\mathrm{b}_{1}} \\
F_{\mathrm{t}_{1}}
\end{array}\right), \\
& M_{1}=\left(\begin{array}{c}
M_{\mathrm{p}_{1}} \\
M_{\mathrm{b}_{1}} \\
M_{\mathrm{t}_{1}}
\end{array}\right)=3\left[T_{2}\left(\begin{array}{c}
M_{\mathrm{p}_{1}} \\
M_{\mathrm{b}_{1}} \\
M_{\mathrm{t}_{1}}
\end{array}\right)\right. \\
& \left.+\left(\begin{array}{c}
r_{2}\left(F_{\mathrm{t}_{1}} \sin \alpha_{2}+F_{\mathrm{b}_{1}} \cos \alpha_{2}\right) \sin \theta_{2} \\
-r_{2}\left(F_{\mathrm{t}_{1}} \sin \alpha_{2}+F_{\mathrm{b}_{1}} \cos \alpha_{2}\right) \cos \theta_{2} \\
r_{2}\left(F_{\mathrm{t}_{1}} \cos \alpha_{1}-F_{\mathrm{b}_{1}} \sin \alpha_{2}\right)
\end{array}\right)\right] \text {. }
\end{aligned}
$$

Finally, the forces and moments of the second stage can be obtained by transforming the components of the triplet forces and moments:

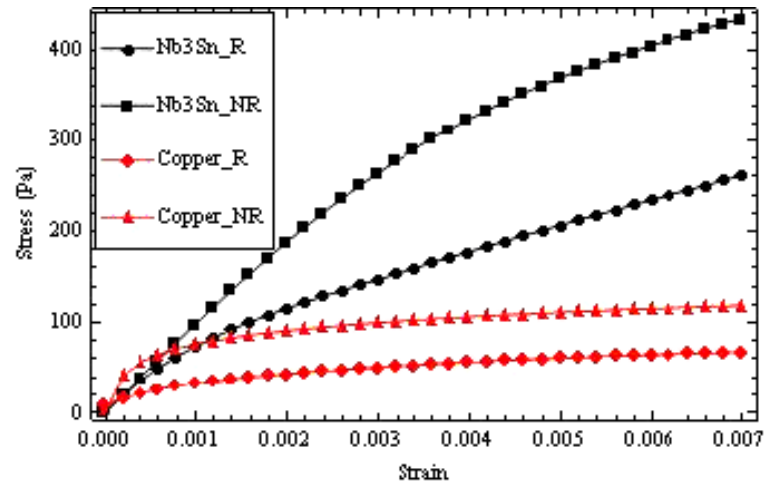

Figure 12. stress-strain curve for $\mathrm{Nb}_{3} \mathrm{Sn}$ and copper wires, R: reacted; NR: not reacted.

$$
\begin{aligned}
F_{3}= & \left(\begin{array}{c}
0 \\
0 \\
F_{\mathrm{t}_{2}}
\end{array}\right)=5 T_{3}\left(\begin{array}{c}
F_{\mathrm{p}_{2}} \\
F_{\mathrm{b}_{2}} \\
F_{\mathrm{t}_{2}}
\end{array}\right), \\
M_{3} & =\left(\begin{array}{c}
0 \\
0 \\
M_{\mathrm{t}_{3}}
\end{array}\right)=3\left[T_{3}\left(\begin{array}{c}
M_{\mathrm{p}_{2}} \\
M_{\mathrm{b}_{2}} \\
M_{\mathrm{t}_{2}}
\end{array}\right)\right. \\
& \left.+\left(\begin{array}{c}
r_{3}\left(F_{\mathrm{t}_{2}} \sin \alpha_{3}+F_{\mathrm{b}_{2}} \cos \alpha_{3}\right) \sin \theta_{3} \\
-r_{3}\left(F_{\mathrm{t}_{2}} \sin \alpha_{3}+F_{\mathrm{b}_{2}} \cos \alpha_{3}\right) \cos \theta_{3} \\
r_{3}\left(F_{\mathrm{t}_{2}} \cos \alpha_{3}-F_{\mathrm{b}_{2}} \sin \alpha_{3}\right)
\end{array}\right)\right],
\end{aligned}
$$

where $F_{\mathrm{t}_{3}}$ and $M_{\mathrm{t}_{3}}$ are the axial force and twist moment applied to the third stage, respectively. Also here the model equation system is nonlinear and the Newton method is applied in the computations to solve it.

\section{Numerical results}

We applied the model to investigate the influence of different superconducting cable layouts on the strain distribution when subjected to axial tensile stress. In that sense the model results may provide useful information for cable design. An overview of most simulation results obtained so far is reported in [23]. Here, we give some results relevant for the initial validation of the model. The materials mechanical properties in terms of axial stress-strain curves of the superconducting $\mathrm{Nb}_{3} \mathrm{Sn}$ and 'normal' copper strands are plotted in figure 12. From the figure, it is easily seen that there is a large difference in 


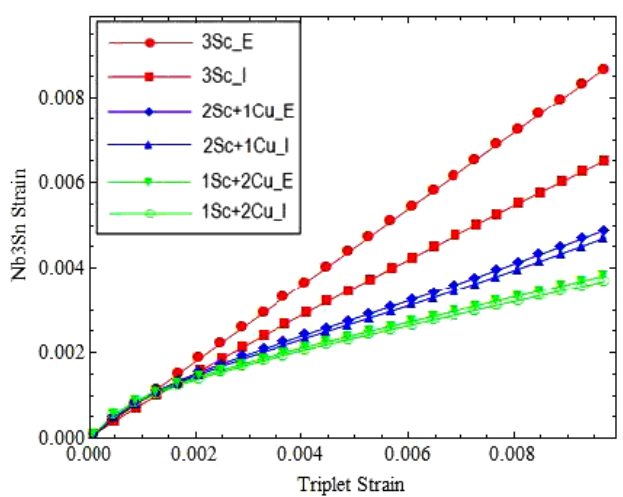

(a)

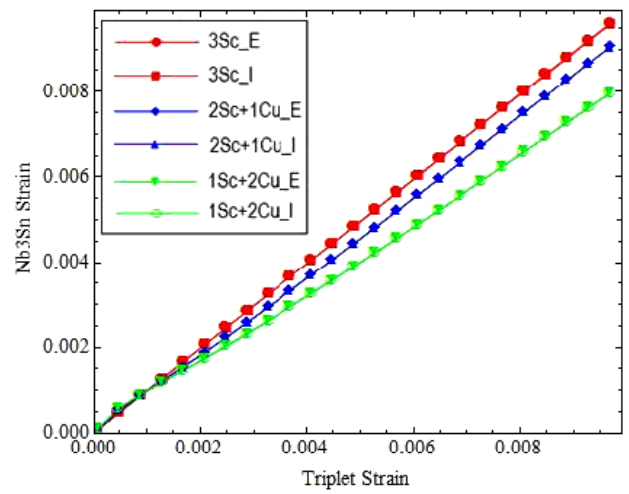

(c)

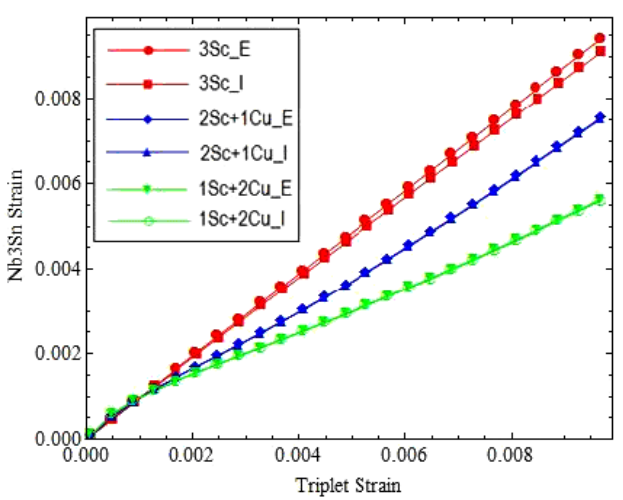

(b)

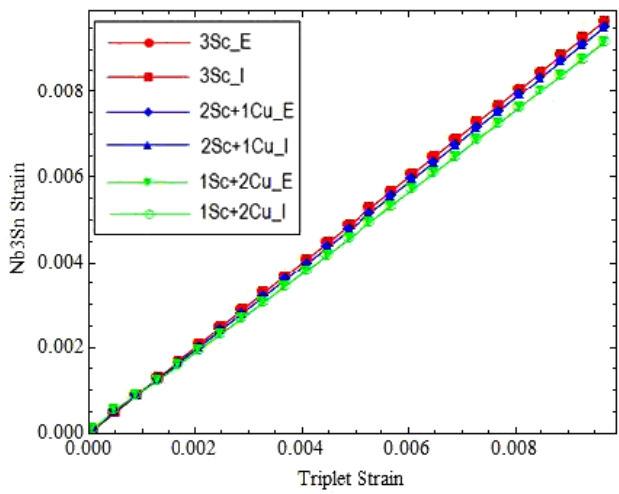

(d)

Figure 13. Wire strain-triplet strain curve for different pitches ((a) pitch $=10 \mathrm{~mm}$, (b) pitch $=20 \mathrm{~mm}$, (c) pitch $=40 \mathrm{~mm}$, (d) pitch $=80 \mathrm{~mm}$ ). E: excluding contact deformation; I: including contact deformation.

behavior between superconducting and copper wire for larger strain.

We start by presenting some triplet model results. The triplet strain is the global applied axial strain on the triplet, while the wire strain is the axial strain (caused by axial force) along the wire axial direction. In figure 13, we see that the $\mathrm{Nb}_{3} \mathrm{Sn}$ wire strain in the triplet with copper wire $(2 \mathrm{Sc}+1 \mathrm{Cu}$ or $1 \mathrm{Sc}+2 \mathrm{Cu}$ ) is much smaller than for a triplet with three superconducting wires $(3 \mathrm{Sc})$, when the applied strain is larger than $0.2 \%$. We also find that the contact force has the largest impact in the triplet with three superconducting wires (3Sc). For long twist pitches (limiting case towards parallel wires), we find that all wire strains are similar. In addition, the contact force between wires gets smaller when the pitch gets longer.

We also compared the model results with an experimental cable stress-strain test for the third stage, but with a cable layout of $3 \times 3 \times 4$, with twist pitches of $26 \mathrm{~mm} \times 70 \mathrm{~mm} \times$ $110 \mathrm{~mm}[22,23]$. The numerical results are plotted in figure 14 .

The material properties of the strands (axial tensile stressstrain curves) are taken from the single wire tests, reported in [22]. For our numerical computations, we have taken the average of the measured strand properties, even if the two behaviors were not so different $\left(\mathrm{Nb}_{3} \mathrm{Sn} 8\right.$ and $\mathrm{Nb}_{3} \mathrm{Sn}$ 9 in figure 14). The comparison between experimental and numerical tests is shown in figure 14 . We find that the numerical model is in good agreement with the experimental results, including the spring-back behavior with released load.

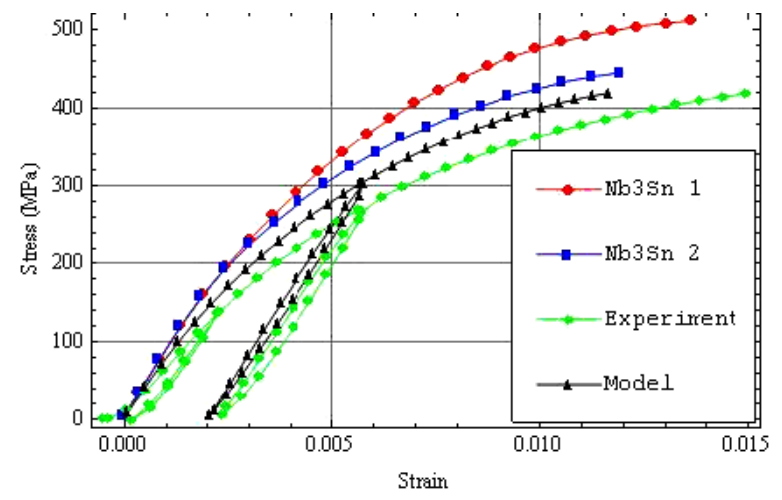

Figure 14. Axial strain versus applied axial stress for stage 3 .

In order to study the influence of the cable layout on the bending strain in strands for a relevant application, we applied the model to the third stage of an ITER TF cable. The cable layout is $3 \times 3 \times 5$, and there is one copper strand in the triplet. We assumed that the global axial strain of stage 3 was $0.5 \%$, and the length was $0.5 \mathrm{~m}$. The maximum bending strains (caused by bending moment or force) and average bending strains are obtained from the model for different pitches. The numerical results are shown in figures 15 and 16 .

The results of the model computations show clearly that the twist pitch has a large influence on the maximum and average bending strain occurring in the single wire, especially 


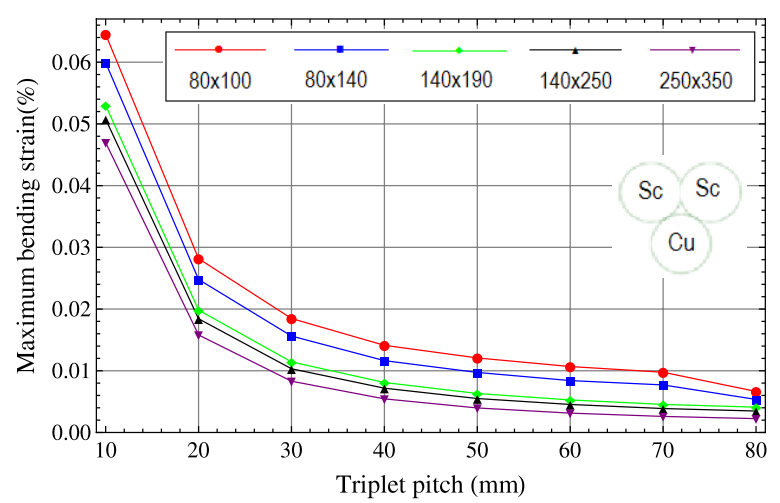

Figure 15. Maximum bending strains for single strands of different layouts for stage 3 (the legend indicates the twist pitches of the second and third stages).

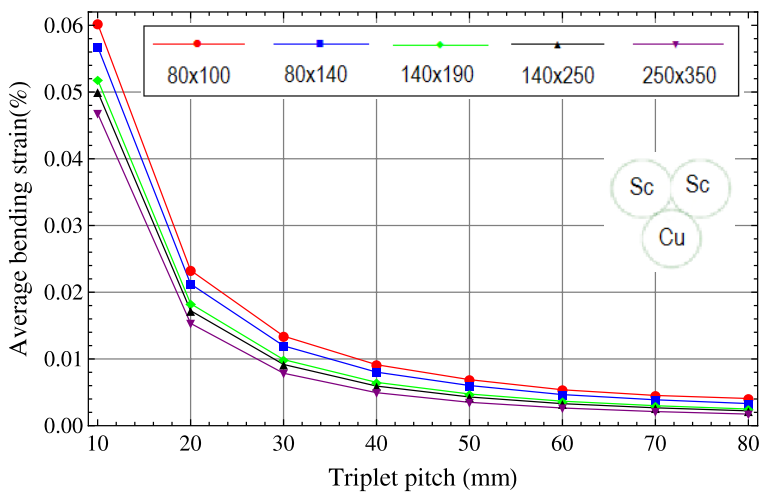

Figure 16. Average bending strains for single strands of different layouts for stage 3 (the legend indicates the twist pitches of the second and third stages).

for small values of the first stage triplet pitch. But also for longer triplet twist pitch above, for instance, $50 \mathrm{~mm}$, the higher stages twist pitch length has a significant influence on the occurring bending strain. Altogether, it appears that the application of short pitches causes significant deformation and large peak bending strains in the strands. When we assume that the tensile axial stress-strain test of a cable is largely representative of the behavior in operation, it can be stated that the application of longer pitches for $\mathrm{Nb}_{3} \mathrm{Sn}$ cables is favorable compared to relatively shorter and intermediate ones.

For stage 3, we also compared different ITER TF cable layouts as tested in SULTAN. One cabling pattern is the socalled Option-II with $(80 \times 140 \times 190) \mathrm{mm}$ pitches [24], which is used for the conductors in the ITER TF coils. We also looked at the 'Long Pitch' used in the TFPRO2-OST2 sample with $(116 \times 182 \times 245) \mathrm{mm}$ pitches [3, 25, 27], the previous TF design $($ Option-I) with $(45 \times 85 \times 125) \mathrm{mm}$ pitches [25], and the recently tested EUTF5-ENEA leg with $(85 \times 135 \times 200)$ $\mathrm{mm}$ pitches and EUTF5-Nexan leg with slightly deviating $(90 \times 140 \times 250) \mathrm{mm}$ pitches. In figure 17 , it is clearly shown that the single strand bending strain in the Option-I cabling pattern becomes relatively high, while the Long Pitch pattern leads to the lowest bending strains. The Option-II pattern leads to significantly lower bending strain than Option-I. The small variations between the EUTF5-ENEA and EUTF5-Nexans

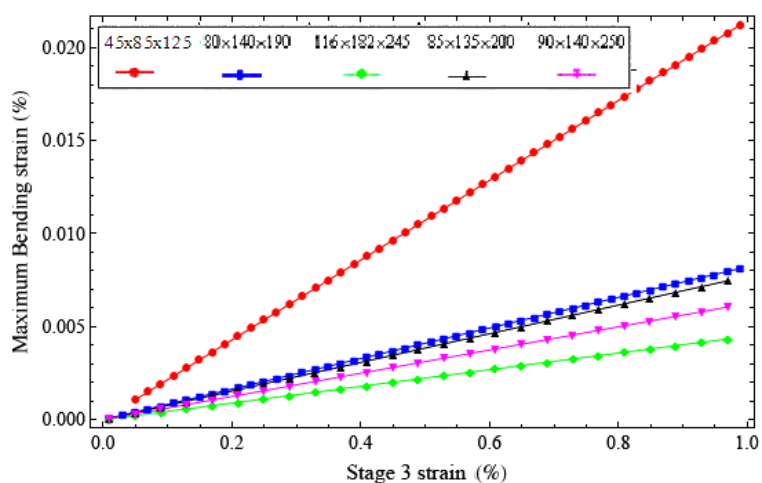

Figure 17. Bending strain for a single strand, under different axial strains of stage 3 .

legs still correspond to the sequence in cabling pitches and a corresponding difference in $T_{\mathrm{cs}}$ is experimentally observed.

The CORD modeling results presented in this paper are well in line with the predictions presented and published in 2006 [25] and experimentally validated a few years later [26, 27]. Moreover, all the experiments using fullsize and sub-size CICCs with varying twist pitch patterns performed in the experiments presented in $[3,22,26,27]$ are in agreement. However, it remains desirable to extend the work, not only in the sense of experimental validation but also in the direction of more detailed modeling (full-size ITER CICC and compaction).

\section{Conclusions}

The novel cable model (CORD) is able to simulate the strain distribution in the individual strands of a multi-stage cabled CICC for an axial tensile strain test, including contact force. Numerical simulations of various cabling stages with different layouts and pitches are presented, up to the third stage. A first validation of the model is performed with a comparison to an experimental third stage cable stress-strain test. The present model can be used to analyze controlled mechanical behavior during cabling and selecting the optimized strand tensions. Assuming that the strand contact distribution and possible strand deflection are relevant for thermal contraction during cool down and transverse electromagnetic forces, the computed strand strain distribution is useful for conductor performance evaluation in relation to the cable pattern. The model shows that longer pitches cause a reduction of $\mathrm{Nb}_{3} \mathrm{Sn}$ strand bending and shear strains, which improves the performance and allows a larger temperature margin.

\section{Acknowledgment}

The support by the IMPACT institute at the University of Twente is greatly acknowledged.

\section{References}

[1] ITER Structural Design Criteria for Magnet Components (SDC-MC) 2001 N11 FDR 5001-07-05 R 0.1 NAKA, Japan 
[2] 2004 ITER Final Design Report IAEA Vienna and ITER IT team Design Description Document 1.1 Update January

[3] Nijhuis A 2008 A solution for transverse load degradation in ITER $\mathrm{Nb}_{3}$ Sn CICCs: verification of cabling effect on Lorentz force response Supercond. Sci. Technol. 21054011

[4] van den Eijnden N C and Nijhuis A 2005 Axial tensile stress-strain characterization of ITER model coil type $\mathrm{Nb}_{3} \mathrm{Sn}$ strands in TARSIS Supercond. Sci. Technol. 18 1-10

[5] Costello G A 1997 Theory of Wire Rope 2nd edn (Berlin: Springer)

[6] Love A E H 1944 A Treatise on the Mathematical Theory of Elasticity (New York: Dover)

[7] Phillips J W and Costello G A 1979 Gernal axial response of stranded wire helical springs Int. J. Non-Linear Mech. 14 247-57

[8] Costello G A and Phillips J W 1979 Static response of stranded wire helical springs Int. J. Non-Linear Mech. 21 171-81

[9] Velinsky S A 1985 General nonlinear theory for complex wire rope Int. J. Mech. Sci. 27 497-507

[10] Lee W K 1991 An insight into wire rope geometry Int. J. Solids Struct. 28 471-90

[11] Raoof M and Hobbs R E 1988 Analysis of multilayered structurestrands J. Eng. Mech., ASCE 114 1166-82

[12] Yen J and Chen C 2006 Theoretical approach to the solutions of axially loaded complex ropes J. Chin. Inst. Eng. 29 695-701

[13] Feyrer K 2007 Wire Ropes: Tension, Endurance, Reliability (Berlin: Springer)

[14] Kruijer M 2006 Modelling the time-dependent mechanical behaviour of steel reinforced thermo-plasticpipes $P h D$ Thesis

[15] Elata D, Eshkenazy R and Weiss M P 2004 The mechanical behaviour of a wire rope with an indepen-dent wire rope core Int. J. Solids Struct. 41 1157-72
[16] Nawrocki A and Labrosse M 2000 A fnite element model for simple straight wire rope strands Comput. Struct. 77 345-59

[17] Kusy R P and Dilley G J 1984 Elastic modulus of a triple-strand stainless steel arch wire via three-and four-point bending J. Dent. Res. 63 1232-40

[18] Inagaki K, Ekh J and Zahrai S 2007 Mechanical analsis of second order helical structure in electrical cable Int. J. Solids Struct. 44 1657-79

[19] Nemov A S et al Generalized stiffness coefcients for ITER superconducting cables, direct FE modeling and initial confguration Cryogenics 50 304-13

[20] Johnson K L 1985 Contact Mechanics (Cambridge: Cambridge University Press)

[21] Gere J M and Timoshenko S P 1999 Mechanics of Materials (Cheltenham: Stanley Thornes Ltd)

[22] Ilyin Y, Nijhuis A, Wessel W A J, van den Eijnden N and ten Kate H H J 2006 Axial tensile stress-strain characterization of $36 \mathrm{Nb}_{3}$ Sn strands cable IEEE Trans. Appl. Supercond. 16 1249-52

[23] Qin J, Warnet L L, Wu Y and Nijhuis A 2008 CORD, a novel numerical mechanical model for $\mathrm{Nb}_{3}$ Sn CICCs IEEE Trans. Appl. Supercond. 18 1105-8

[24] Besi Vetrella U et al 2008 Manufacturing of the the ITER TF full size prototype conductor IEEE Trans. Appl. Supercond. 18 1105-8

[25] Nijhuis A and Ilyin Y 2006 Transverse load optimization in $\mathrm{Nb}_{3} \mathrm{Sn}$ CICC design; influence of cabling, void fraction and strand stiffness Supercond. Sci. Technol. 19 945-62

[26] Bruzzone $\mathrm{P}$ et al 2009 Test results of a $\mathrm{Nb}_{3}$ Sn cable-in-conduit conductor with variable pitch sequence IEEE Trans. Appl. Supercond. 19 1448-51

[27] Bessette D and Mitchell N 2008 Review of the results of the ITER toroidal field conductor R\&D and qualification IEEE Trans. Appl. Supercond. 18 1109-13 